\title{
Breakdown of the Stokes-Einstein Equation for Solutions of Water in Oil Reverse Micelles
}

Markus M. Hoffmann, ${ }^{* 1}$ Matthew D. Too, ${ }^{1}$ Michael Vogel, ${ }^{2}$ Torsten Gutmann, ${ }^{3}$ and Gerd Buntkowsky*3

${ }^{1}$ Department of Chemistry and Biochemistry, State University of New York College at Brockport, Brockport, NY, 14420, USA

${ }^{2}$ Institute of Condensed Matter Physics, Technical University Darmstadt, Hochschulstraße 6, 64289 Darmstadt, Germany

${ }^{3}$ Institute of Physical Chemistry, Technical University Darmstadt, Alarich-Weiss-Straße 8, D-64287 Darmstadt, Germany

Corresponding authors: $\quad$ mhoffman@brockport.edu

gerd.buntkowsky@chemie.tu-darmstadt.de 
Table S1. Composition of solutions of $15 \%$ by mass $C_{10} E_{6}$ in cyclohexane with indicated water content for NMR, viscosity, and density measurements

\begin{tabular}{rrrrrr}
\hline$\% \mathrm{H}_{2} \mathrm{O}$ & $\mathrm{C}_{10} \mathrm{E}_{6} / \mathrm{g}$ & cyclohexane/g & water $/ \mathrm{g}$ & $\mathrm{m} / \mathrm{mol} \mathrm{kg}^{-1}$ & $W^{\mathrm{a}}$ \\
\hline 0 & 1.4924 & 8.6420 & 0.0050 & 0.398 & 0.08 \\
1 & 1.4906 & 8.5339 & 0.1067 & 0.402 & 1.73 \\
2 & 1.4864 & 8.7155 & 0.2046 & 0.393 & 3.32 \\
3 & 1.4951 & 8.5042 & 0.3099 & 0.405 & 5.00 \\
4 & 1.4795 & 8.5445 & 0.3911 & 0.399 & 6.37 \\
3 & $0.1366^{\mathrm{b}}$ & 0.7808 & 0.0277 & 0.378 & 4.56 \\
\hline
\end{tabular}

${ }^{a} W=\left[\mathrm{H}_{2} \mathrm{O}\right] /\left[\mathrm{C}_{10} \mathrm{E}_{6}\right]$

${ }^{\mathrm{b}}$ monodisperse $\mathrm{C}_{10} \mathrm{E}_{6}$

Table S2. Viscosities in $\mathrm{mPa} \cdot \mathrm{s}$ of solutions of $15 \%$ by mass polydisperse $\mathbf{C}_{10} \mathbf{E}_{6}$ in cyclohexane with indicated water content

\begin{tabular}{ccccccc}
\hline $\mathrm{T} /{ }^{\circ} \mathrm{C} \% \mathrm{H}_{2} \mathrm{O}$ & 0 & 1 & 2 & 3 & \multicolumn{2}{c}{$4^{\mathrm{a}}$} \\
$W$ & 0.08 & 1.73 & 3.32 & 5.00 & 6.37 & - \\
\hline 10 & 1.766 & 1.865 & 2.053 & 3.534 & - & - \\
15 & 1.550 & 1.667 & 1.823 & 2.845 & 9.022 & 8.785 \\
20 & 1.384 & 1.498 & 1.629 & 2.371 & 7.071 & 6.656 \\
25 & 1.259 & 1.350 & 1.461 & 1.993 & 5.103 & 5.422 \\
30 & 1.131 & 1.220 & 1.317 & 1.709 & 3.806 & 3.891 \\
35 & 1.027 & 1.107 & 1.191 & 1.483 & 2.880 & 2.876 \\
40 & 0.937 & 1.007 & 1.081 & 1.300 & 2.283 & 2.188 \\
45 & 0.858 & 0.919 & 0.983 & 1.146 & 1.797 & 1.706 \\
\hline
\end{tabular}

a from two independently prepared samples

Table S3. Viscosity standard deviations in $\mathrm{mPa} \cdot \mathrm{s}$ of solutions of $15 \%$ by mass polydisperse $\mathbf{C}_{10} E_{6}$ in cyclohexane with indicated water content

\begin{tabular}{ccccccc}
$\mathrm{T} /{ }^{\circ} \mathrm{Cl} / \mathrm{H}_{2} \mathrm{O}$ & 0 & 1 & 2 & 3 & \multicolumn{2}{c}{$4^{\mathrm{a}}$} \\
$W$ & 0.08 & 1.73 & 3.32 & 5.00 & 6.37 \\
\hline 10 & 0.004 & 0.001 & 0.001 & 0.002 & - & - \\
15 & 0.003 & 0.001 & 0.001 & 0.002 & 0.004 & 0.002 \\
20 & 0.000 & 0.001 & 0.001 & 0.019 & 0.002 & 0.004 \\
25 & 0.005 & 0.002 & 0.001 & 0.003 & 0.006 & 0.101 \\
30 & 0.002 & 0.002 & 0.002 & 0.004 & 0.007 & 0.027 \\
35 & 0.002 & 0.002 & 0.003 & 0.004 & 0.004 & 0.010 \\
40 & 0.003 & 0.003 & 0.004 & 0.005 & 0.016 & 0.008 \\
45 & 0.004 & 0.005 & 0.005 & 0.004 & 0.014 & 0.006 \\
\hline
\end{tabular}

${ }^{\mathrm{a}}$ from two independently prepared samples 


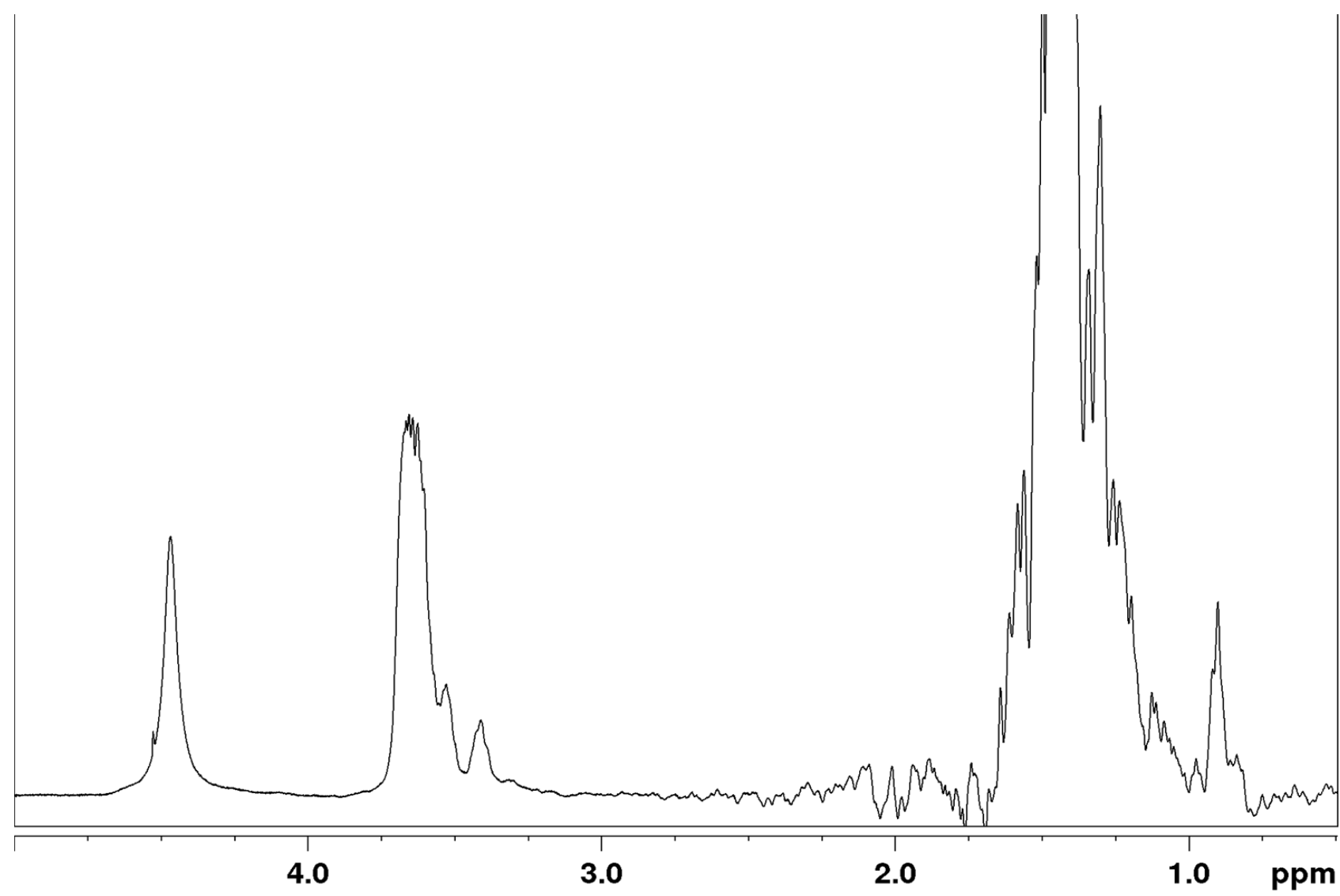

Figure S1. Proton spectrum for $15 \%$ by mass polydisperse $\mathrm{C}_{10} \mathrm{E}_{6}$ in cyclohexane with three mass percent water at $15^{\circ} \mathrm{C}$. The spectral assignment is as follows. Due to fast chemical exchange, only one peak is observed for water and hydroxyl protons near a chemical shift near $4.5 \mathrm{ppm}$. Protons from the ethylene oxide repeat unit are observable as peaks near 3.7, and 3.6, ppm. The peak at $3.4 \mathrm{ppm}$ is from the methylene protons of the alkyl chain in $\alpha$-position to the ethylene oxide repeat unit. The cut off large peak near $1.4 \mathrm{ppm}$ is from cyclohexane, and it overlaps with the methylene protons of the alkyl chain. The terminal alkyl chain methyl protons are reflected by the peak at $0.9 \mathrm{ppm}$. Chemical shifts for these peaks were dependent on sample temperature, in particular for the water/hydroxyl peak, but the relative chemical shift order did not change. 


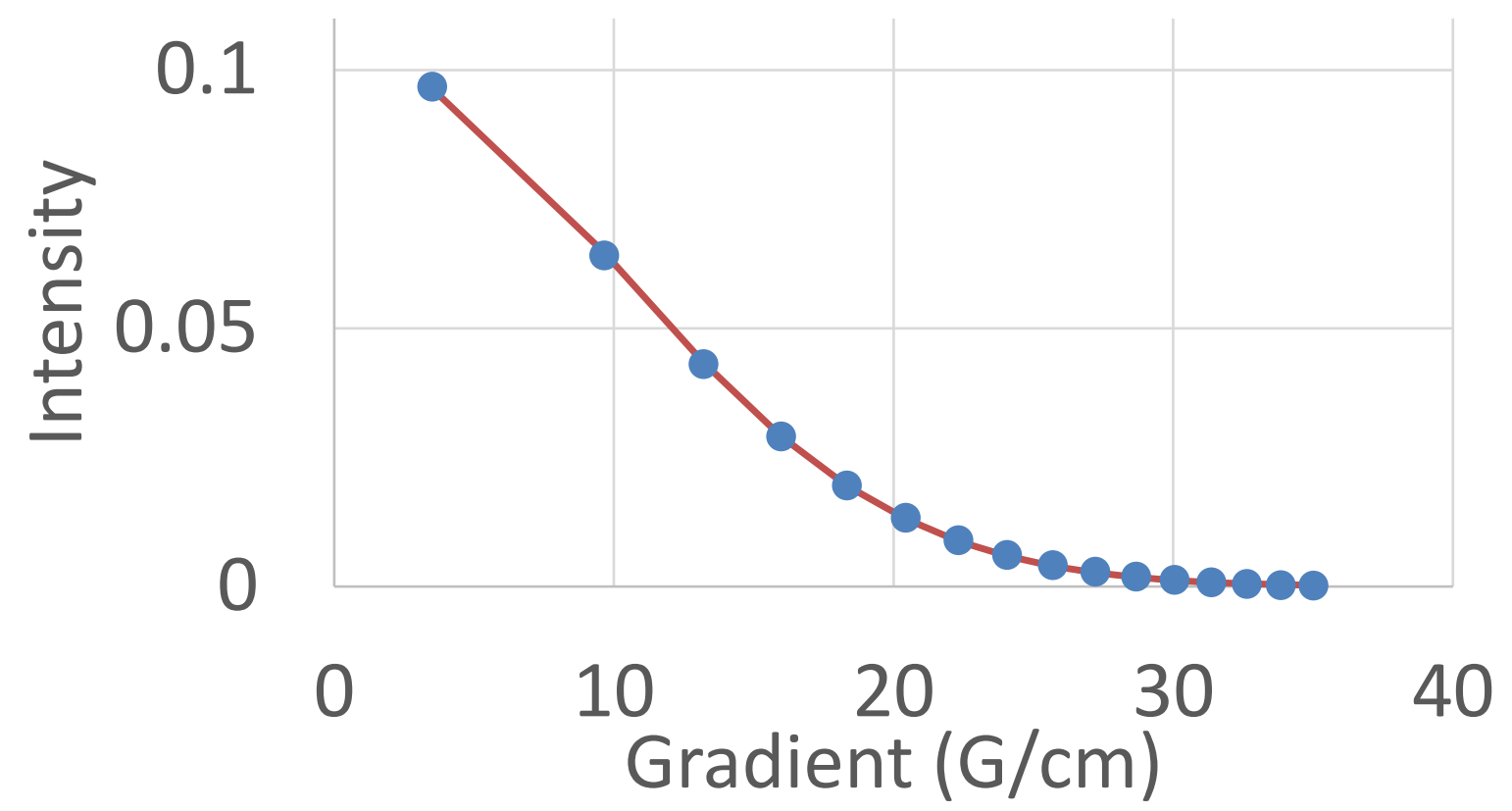

Figure S2. Proton resonance intensity of ethylene oxide repeat unit as a function of gradient strength for the sample of $15 \%$ by mass polydisperse $\mathrm{C}_{10} \mathrm{E}_{6}$ in cyclohexane at $35^{\circ} \mathrm{C}$ without any added water.

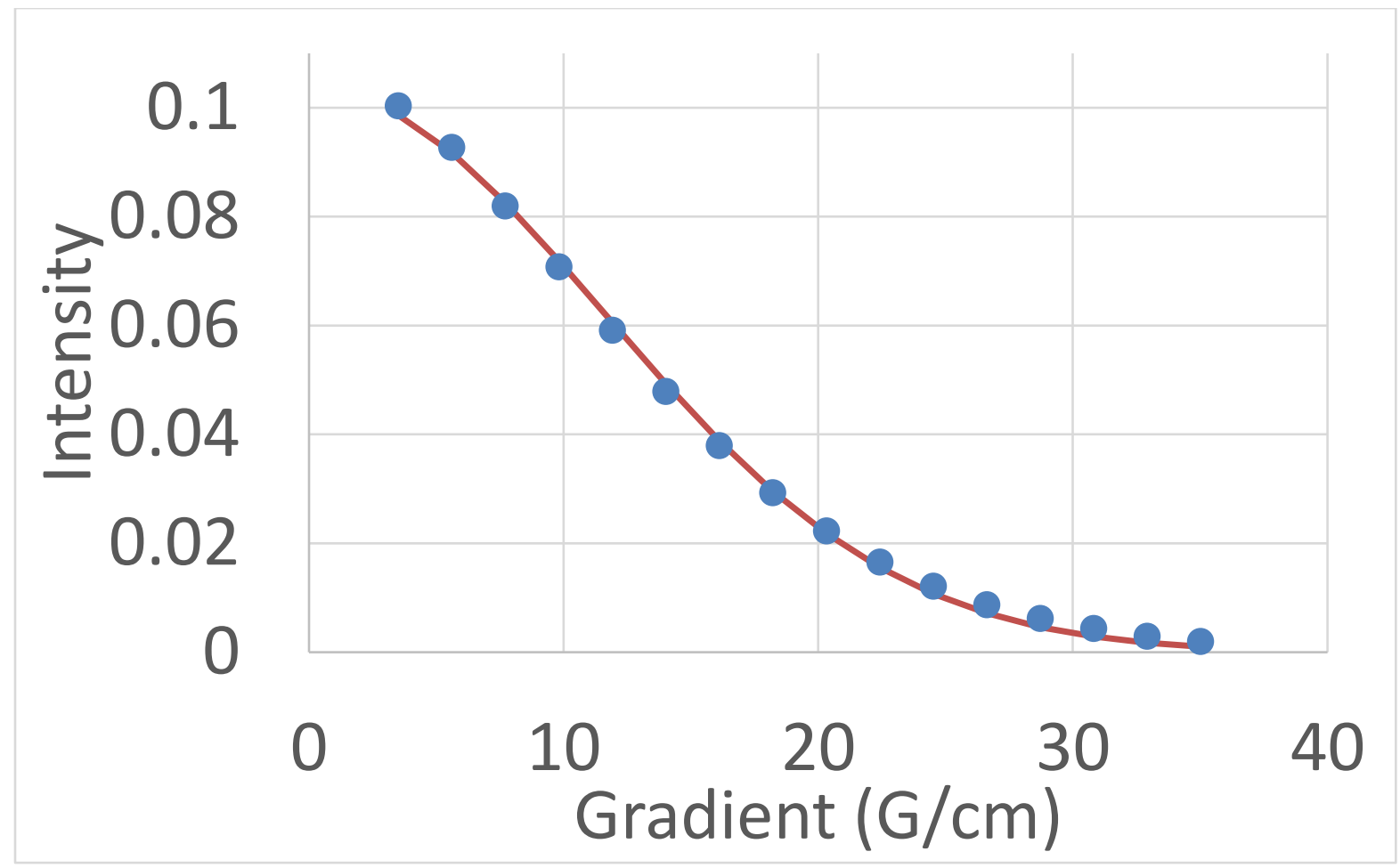

Figure S3. Proton resonance intensity of ethylene oxide repeat unit as a function of gradient strength for the sample of $15 \%$ by mass polydisperse $\mathrm{C}_{10} \mathrm{E}_{6}$ in cyclohexane at $35^{\circ} \mathrm{C}$ with $1 \%$ by mass added water. 


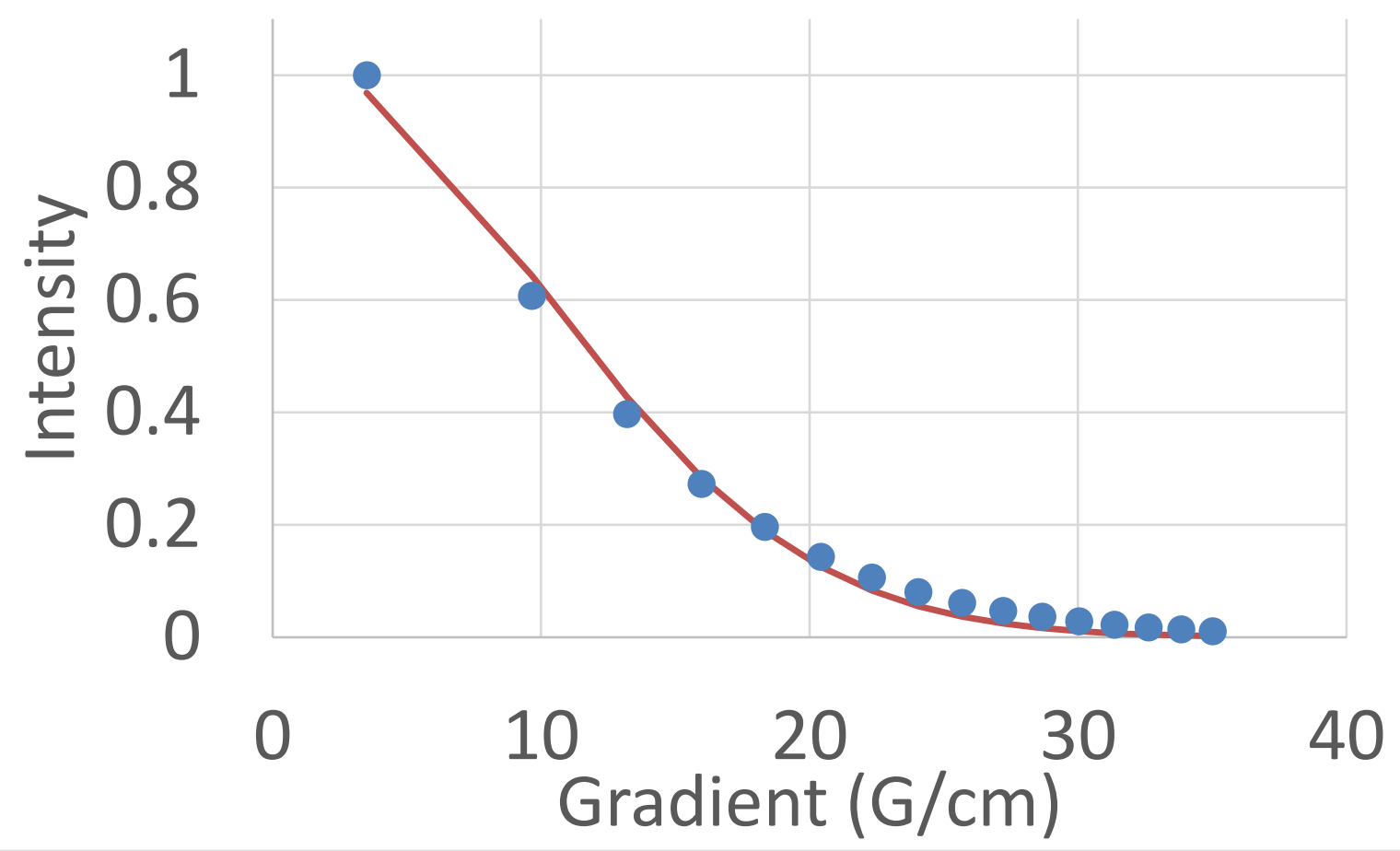

Figure S4. Proton resonance intensity of ethylene oxide repeat unit as a function of gradient strength for the sample of $15 \%$ by mass polydisperse $\mathrm{C}_{10} \mathrm{E}_{6}$ in cyclohexane at $35^{\circ} \mathrm{C}$ with $2 \%$ by mass added water.

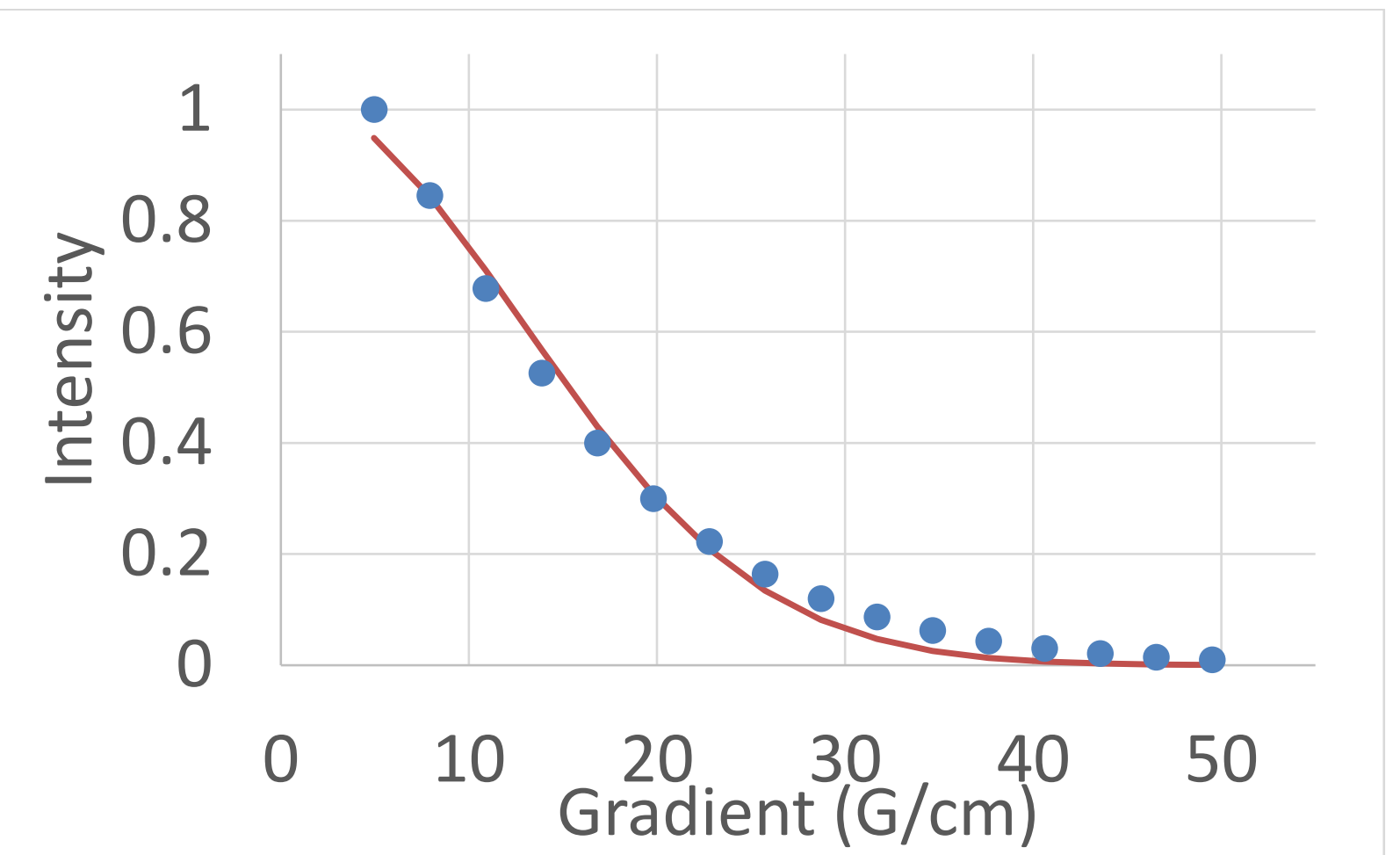

Figure S5. Proton resonance intensity of ethylene oxide repeat unit as a function of gradient strength for the sample of $15 \%$ by mass polydisperse $\mathrm{C}_{10} \mathrm{E}_{6}$ in cyclohexane at $35^{\circ} \mathrm{C}$ with $3 \%$ by mass added water. 


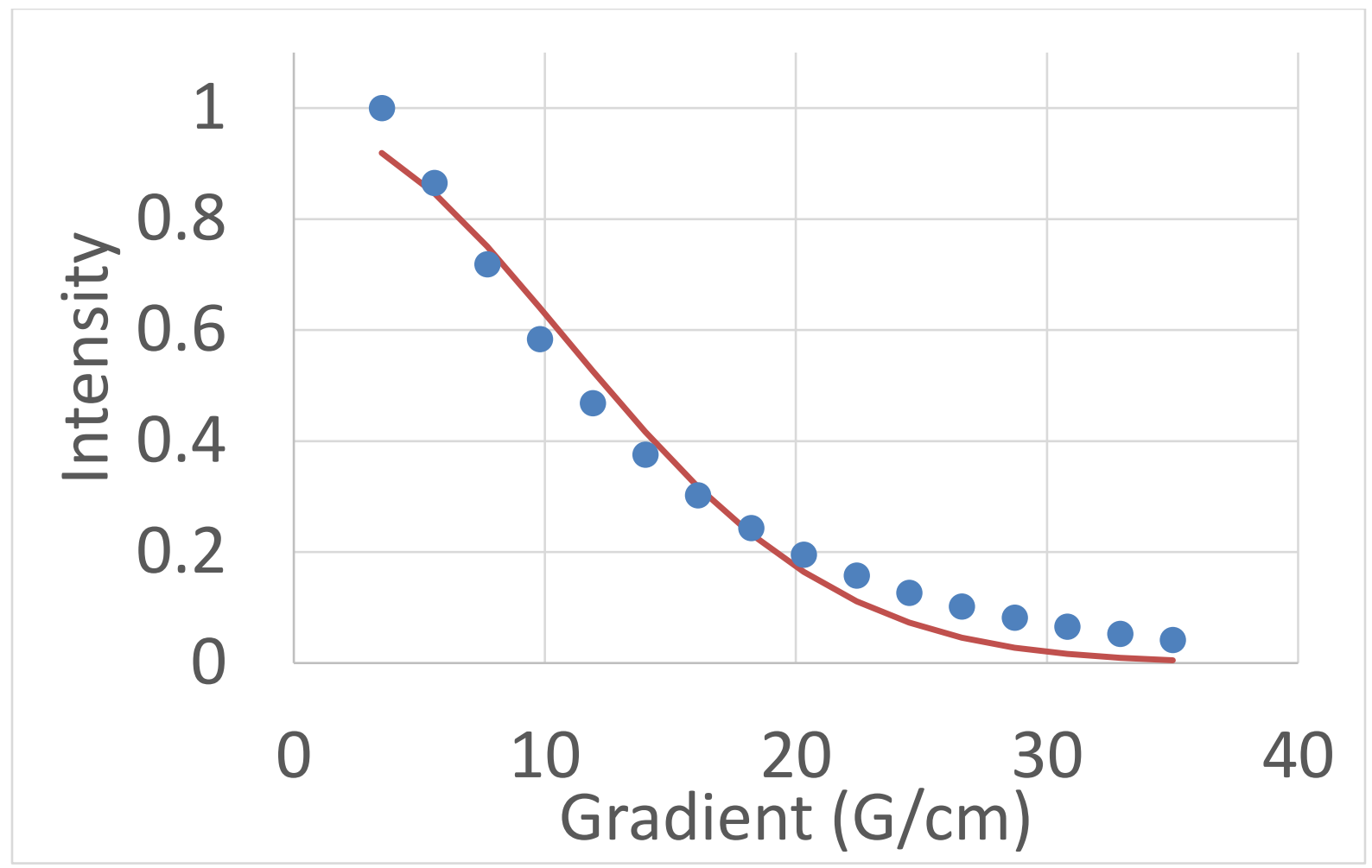

Figure S6. Proton resonance intensity of ethylene oxide repeat unit as a function of gradient strength for the sample of $15 \%$ by mass polydisperse $\mathrm{C}_{10} \mathrm{E}_{6}$ in cyclohexane at $35^{\circ} \mathrm{C}$ with $4 \%$ by mass added water. 


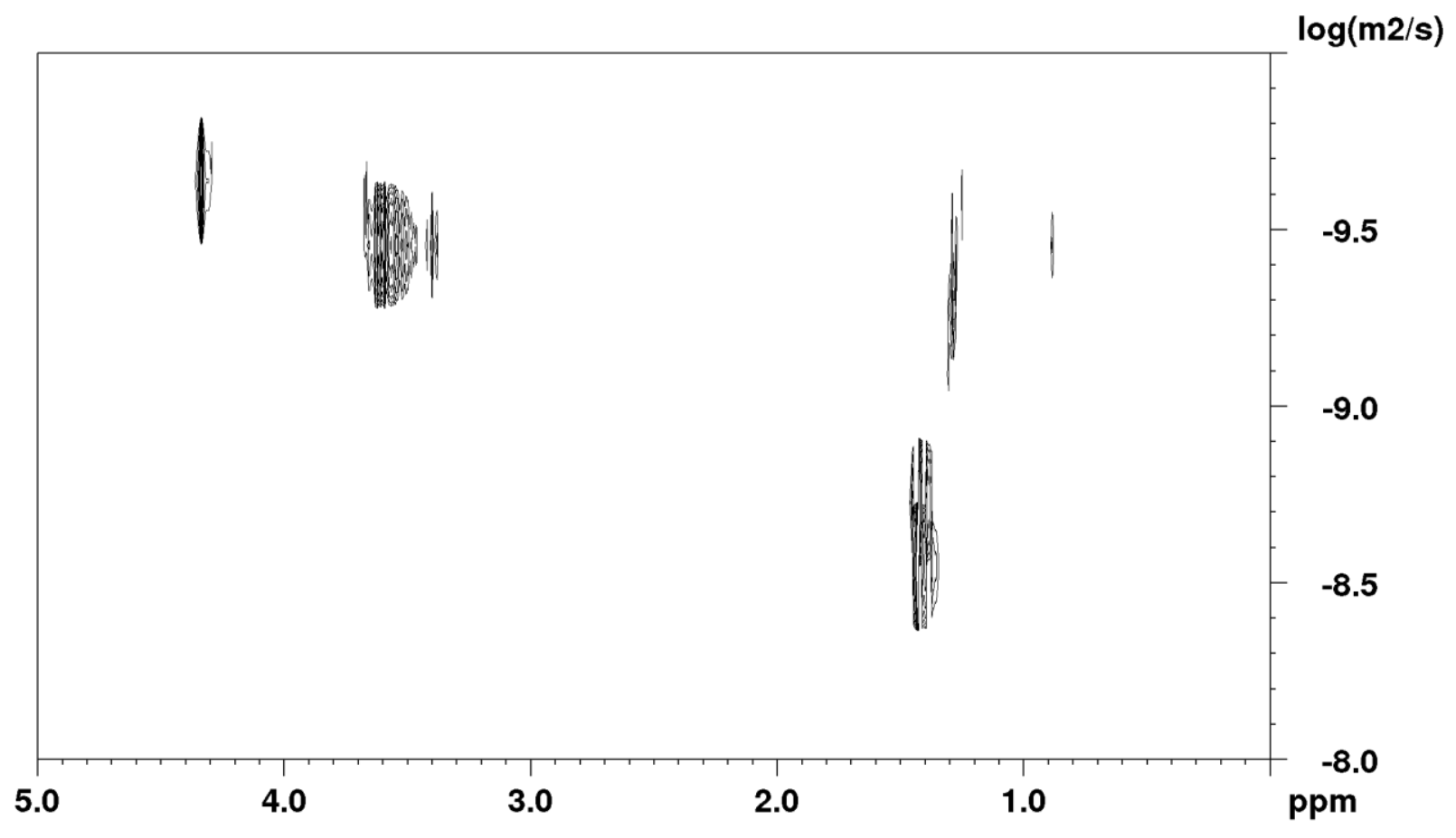

Figure S7. Two-dimensional DOSY spectrum for $15 \%$ by mass monodisperse $\mathrm{C}_{10} \mathrm{E}_{6}$ in cyclohexane with three mass percent water at $45^{\circ} \mathrm{C}$ showing diffusion relations among components. 
Table S4. $\mathrm{C}_{10} \mathrm{E}_{6}$ Solute self-diffusion coefficients in $10^{-10} \mathrm{~m}^{2} \cdot \mathrm{s}^{-1}$ in solutions of $15 \%$ by mass $\mathrm{C}_{10} \mathrm{E}_{6}$ in cyclohexane with indicated water content

\begin{tabular}{crrrrrr}
\hline $\mathrm{T} /{ }^{\circ} \mathrm{C} \% \mathrm{H}_{2} \mathrm{O}$ & 0 & 1 & 2 & 3 & 4 & $3 *$ \\
$W$ & 0.08 & 1.73 & 3.32 & 5.00 & 6.37 & 4.56 \\
\hline 10 & 2.09 & 1.25 & 0.86 & 0.56 & 0.38 & \\
15 & 2.26 & 1.36 & 1.07 & 0.60 & 0.55 & \\
20 & 2.66 & 1.71 & 1.32 & 0.87 & 0.51 & \\
25 & 3.16 & 1.97 & 1.47 & 0.91 & 0.80 & 1.22 \\
& & & & & 0.76 & \\
30 & 3.51 & 2.35 & 1.66 & 1.31 & 0.84 & \\
35 & 4.27 & 2.85 & 2.23 & 1.60 & 1.19 & 1.93 \\
& & 2.84 & 2.18 & 1.65 & 1.21 & \\
40 & & 2.84 & 2.14 & 1.76 & 1.25 & \\
45 & 4.83 & 3.44 & 2.70 & 1.87 & 1.72 & \\
*sample from monodisperse $\mathrm{C}_{10} \mathrm{E}_{6}$ & 4.24 & 3.24 & 2.53 & 2.28 & 2.91 \\
\hline
\end{tabular}

*sample from monodisperse $\mathrm{C}_{10} \mathrm{E}_{6}$

Table S5. Standard deviations of $\mathrm{C}_{10} \mathrm{E}_{6}$ solute self-diffusion coefficients in $10^{-10} \mathrm{~m}^{2} \cdot \mathrm{s}^{-1}$ in solutions of $15 \%$ by mass $C_{10} E_{6}$ in cyclohexane with indicated water content

\begin{tabular}{crrrrrr}
\hline $\mathrm{T} /{ }^{\circ} \mathrm{Cl} \% \mathrm{H}_{2} \mathrm{O}$ & 0 & 1 & 2 & 3 & 4 & $3 *$ \\
$W$ & 0.08 & 1.73 & 3.32 & 5.00 & 6.37 & 4.56 \\
\hline 10 & 0.25 & 0.20 & 0.18 & 0.27 & 0.25 & \\
15 & 0.18 & 0.21 & 0.24 & 0.20 & 0.24 & \\
20 & 0.22 & 0.26 & 0.29 & 0.26 & 0.18 & \\
25 & 0.30 & 0.31 & 0.29 & 0.27 & 0.29 & 0.04 \\
& & & & & 0.28 & \\
30 & 0.29 & 0.29 & 0.30 & 0.36 & 0.32 & \\
35 & 0.66 & 0.34 & 0.46 & 0.39 & 0.43 & 0.03 \\
& & 0.41 & 0.44 & 0.46 & 0.36 & \\
40 & & 0.39 & 0.42 & 0.47 & 0.37 & \\
45 & 0.49 & 0.43 & 0.69 & 0.49 & 0.66 & \\
& 0.75 & 0.55 & 0.96 & 0.66 & 0.68 & 0.11 \\
\hline
\end{tabular}

*sample from monodisperse $\mathrm{C}_{10} \mathrm{E}_{6}$ 
Table S6. Cyclohexane solvent self-diffusion coefficients in $10^{-10} \mathrm{~m}^{2} \cdot \mathrm{s}^{-1}$ in solutions of $15 \%$ by mass $\mathrm{C}_{10} \mathrm{E}_{6}$ in cyclohexane with indicated water content

\begin{tabular}{crrrrrr}
\hline $\mathrm{T} /{ }^{\circ} \mathrm{C} \% \mathrm{H}_{2} \mathrm{O}$ & 0 & 1 & 2 & 3 & 4 & $3^{*}$ \\
$W$ & 0.08 & 1.73 & 3.32 & 5.00 & 6.37 & 4.56 \\
\hline 10 & 8.8 & 9.1 & 9.1 & 8.6 & 8.8 & \\
15 & 9.9 & 9.9 & 10.1 & 9.9 & 9.7 & \\
20 & 10.8 & 11.2 & 11.2 & 10.7 & 10.8 & \\
25 & 11.4 & 12.0 & 12.0 & 11.9 & $11.6^{\mathrm{a}}$ & 12.78 \\
30 & 12.7 & 13.3 & 13.7 & 13.1 & 13.0 & \\
35 & 14.2 & $14.6^{\mathrm{b}}$ & $15.0^{\mathrm{b}}$ & $14.5^{\mathrm{b}}$ & $14.5^{\mathrm{c}}$ & 14.92 \\
40 & 15.7 & 15.9 & 15.9 & 15.4 & 16.1 & \\
45 & 17.1 & 18.2 & 16.9 & 17.5 & 17.6 & 17.96 \\
\hline
\end{tabular}

Average of $\mathrm{two}^{\mathrm{a}}$, three ${ }^{\mathrm{b}}$, and seven ${ }^{\mathrm{c}}$ DOSY measurements. Relative standard deviations of measurements are estimated to be less than $2 \%$ based on repeated measurements of select samples.

*sample from monodisperse $\mathrm{C}_{10} \mathrm{E}_{6}$

Table S7. Water self-diffusion coefficients in $10^{-10} \mathrm{~m}^{2} \cdot \mathrm{s}^{-1}$ in solutions of $15 \%$ by mass $\mathrm{C}_{10} \mathrm{E}_{6}$ in cyclohexane with indicated water content

\begin{tabular}{crrrrr}
\hline $\mathrm{T} /{ }^{\circ} \mathrm{Cl} \% \mathrm{H}_{2} \mathrm{O}$ & 1 & 2 & 3 & 4 & $3 *$ \\
$W$ & 0.08 & 1.73 & 3.32 & 5.00 & 6.37 \\
\hline 10 & 1.46 & 0.58 & 0.34 & 0.43 & \\
15 & 1.40 & 0.65 & 0.44 & 0.55 & \\
20 & 1.66 & 0.82 & 0.57 & 0.67 & \\
25 & 1.94 & 0.95 & 0.65 & 0.70 & 0.77 \\
30 & 2.15 & 1.22 & 0.87 & 0.89 & \\
35 & 2.63 & 1.54 & 1.08 & 1.02 & 1.18 \\
40 & 3.20 & 1.94 & 1.29 & 1.24 & \\
45 & 3.59 & 2.46 & 1.63 & 1.49 & 1.73 \\
\hline
\end{tabular}

Relative standard deviations of measurements are estimated to be less than $10 \%$.

*sample from monodisperse $\mathrm{C}_{10} \mathrm{E}_{6}$ 
Table S8. Average $\mathrm{C}_{10} \mathrm{E}_{6}$ speciation radii (in $\AA$ ) generated by the

Stokes-Einstein equation (eqs 1 and 4 ) for solutions of $15 \%$ by mass polydisperse $\mathrm{C}_{10} \mathrm{E}_{6}$ in cyclohexane with indicated water content $\mathrm{T} /{ }^{\circ} \mathrm{Cl} \% \mathrm{H}_{2} \mathrm{O}$

$\begin{array}{llll}1 & 2 & 3 & 4\end{array}$

\begin{tabular}{rrrrrr}
$W$ & 0.08 & 1.73 & 3.32 & 5.00 & 6.37 \\
\hline 10 & 6.3 & 9.4 & 12.1 & 10.9 & $-^{\mathrm{a}}$ \\
15 & 6.7 & 9.7 & 11.2 & 12.7 & 5.1 \\
20 & 6.5 & 8.9 & 10.4 & 10.8 & 6.6 \\
25 & 6.2 & 8.7 & 10.6 & 12.3 & 6.2 \\
30 & 6.3 & 8.3 & 10.5 & 10.3 & 7.5 \\
35 & 5.9 & 7.7 & 9.1 & 9.6 & 7.0 \\
40 & 5.8 & 7.2 & 8.4 & 9.9 & 6.5 \\
45 & 5.5 & 6.6 & 7.9 & 8.5 & 6.4 \\
\hline
\end{tabular}

${ }^{a}$ no value provided because viscosity was not determined

Table S9. Average $\mathrm{C}_{10} \mathrm{E}_{6}$ speciation radii (in $\AA$ ) generated by ratio of $D_{\mathrm{C} 6 \mathrm{H} 12} / D_{\mathrm{C} 10 \mathrm{E} 6}(\mathrm{eq} 5)$ for solutions of $15 \%$ by mass polydisperse $\mathrm{C}_{10} \mathrm{E}_{6}$ in cyclohexane with indicated water content

\begin{tabular}{crrrrr}
\hline $\mathrm{T} /{ }^{\circ} \mathrm{C} \% \mathrm{H}_{2} \mathrm{O}$ & 0 & 1 & 2 & 3 & 4 \\
$W$ & 0.08 & 1.73 & 3.32 & 5.00 & 6.37 \\
\hline 10 & 12.2 & 21.2 & 30.8 & 44.8 & 67.6 \\
15 & 12.7 & 21.2 & 27.3 & 48.0 & 51.0 \\
20 & 11.8 & 19.0 & 24.6 & 35.5 & 61.7 \\
25 & 10.5 & 17.7 & 23.7 & 37.8 & 43.2 \\
30 & 10.5 & 16.5 & 23.8 & 28.8 & 44.5 \\
35 & 9.7 & 14.9 & 19.8 & 25.2 & 34.5 \\
40 & 9.4 & 13.4 & 17.0 & 23.8 & 27.2 \\
45 & 8.6 & 12.4 & 15.2 & 20.1 & 22.4 \\
\hline
\end{tabular}


Table S10. Uncertainties of the average $C_{10} E_{6}$ speciation radii from the Stokes-Einstein equation (in $\AA$ ) for solutions of $15 \%$ by mass polydisperse $\mathrm{C}_{10} \mathrm{E}_{6}$ in cyclohexane with indicated water content obtained by error propagation

\begin{tabular}{crrrrr}
\hline $\mathrm{T} /{ }^{\circ} \mathrm{Cl} \% \mathrm{H}_{2} \mathrm{O}$ & 0 & 1 & 2 & 3 & 4 \\
$W$ & 0.08 & 1.73 & 3.32 & 5.00 & 6.37 \\
\hline 10 & 0.7 & 1.5 & 2.5 & 5.2 & - \\
15 & 0.5 & 1.5 & 2.5 & 4.3 & 2.2 \\
20 & 0.5 & 1.4 & 2.2 & 3.2 & 2.3 \\
25 & 0.6 & 1.4 & 2.1 & 3.6 & 0.20 \\
30 & 0.5 & 1.0 & 1.9 & 2.8 & 2.9 \\
35 & 0.9 & 0.02 & 0.19 & 0.47 & 0.17 \\
40 & 0.6 & 0.9 & 2.1 & 2.6 & 2.5 \\
45 & 0.7 & 0.9 & 2.3 & 2.2 & 1.9 \\
\hline
\end{tabular}

Table S11. Uncertainties of the average $C_{10} E_{6}$ speciation radii from ratio of $\mathrm{D}_{\mathrm{C} 6 \mathrm{H} 12} / \mathrm{D}_{\mathrm{C} 10 \mathrm{E} 6}$ (in $\AA$ ) for solutions of $15 \%$ by mass polydisperse $C_{10} E_{6}$ in cyclohexane with indicated water content obtained by error propagation

\begin{tabular}{crrrrr}
\hline $\mathrm{T} /{ }^{\circ} \mathrm{C} / \% \mathrm{H}_{2} \mathrm{O}$ & 0 & 1 & 2 & 3 & 4 \\
$W$ & 0.08 & 1.73 & 3.32 & 5.00 & 6.37 \\
\hline 10 & 1.5 & 3.4 & 6.4 & 21.5 & 45 \\
15 & 1.1 & 3.3 & 6.1 & 16.2 & 22 \\
20 & 1.0 & 3.0 & 5.3 & 10.6 & 21 \\
25 & 1.0 & 2.8 & 4.7 & 11.1 & 2 \\
30 & 0.9 & 2.0 & 4.3 & 7.8 & 17 \\
35 & 1.5 & 0.3 & 0.5 & 1.3 & 1 \\
40 & 1.0 & 1.7 & 4.4 & 6.3 & 10 \\
45 & 1.1 & 1.6 & 4.5 & 5.3 & 7 \\
\hline
\end{tabular}

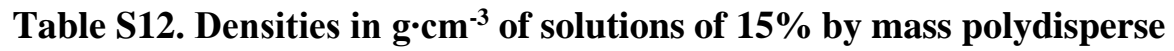
$\mathbf{C}_{10} \mathbf{E}_{6}$ in cyclohexane with indicated water content ${ }^{\mathrm{a}}$

\begin{tabular}{ccccccc}
\hline $\mathrm{T} /{ }^{\circ} \mathrm{C} / \% \mathrm{H}_{2} \mathrm{O}$ & 0 & 1 & 2 & 3 & \multicolumn{2}{c}{$4^{\mathrm{b}}$} \\
$W$ & 0.08 & 1.73 & 3.32 & 5.00 & \multicolumn{2}{c}{6.37} \\
\hline 10 & 0.8117 & 0.8137 & 0.8162 & 0.8179 & 0.8199 & 0.8196 \\
15 & 0.8070 & 0.8091 & 0.8116 & 0.8133 & 0.8153 & 0.8150 \\
20 & 0.8024 & 0.8044 & 0.8069 & 0.8086 & 0.8106 & 0.8103 \\
25 & 0.7977 & 0.7997 & 0.8022 & 0.8039 & 0.8058 & 0.8059 \\
30 & 0.7931 & 0.7950 & 0.7975 & 0.7992 & 0.8011 & 0.8014 \\
35 & 0.7884 & 0.7903 & 0.7928 & 0.7944 & 0.7963 & 0.7967 \\
40 & 0.7837 & 0.7855 & 0.7880 & 0.7896 & 0.7915 & 0.7919 \\
45 & 0.7790 & 0.7808 & 0.7832 & 0.7848 & 0.7867 & 0.7870 \\
\hline
\end{tabular}

${ }^{a}$ Standard deviations from replication are $\leq 0.0001 \mathrm{~g} \cdot \mathrm{cm}^{-3}$

${ }^{\mathrm{b}}$ Measurements from two separately prepared samples to check reproducibility. 
Table S13. Average $\mathrm{C}_{10} \mathrm{E}_{6}$ aggregation numbers using radii generated by the Stokes-Einstein equation for solutions of $15 \%$ by mass polydisperse $\mathrm{C}_{10} \mathrm{E}_{6}$ in cyclohexane with indicated water content

\begin{tabular}{crrrrr}
\hline $\mathrm{T} /{ }^{\circ} \mathrm{C} \% \mathrm{H}_{2} \mathrm{O}$ & 0 & 1 & 2 & 3 & 4 \\
$W$ & 0.08 & 1.73 & 3.32 & 5.00 & 6.37 \\
\hline 10 & 2.3 & 7.2 & 14.5 & 10.0 & - \\
15 & 2.8 & 8.0 & 11.5 & 15.8 & 1.0 \\
20 & 2.6 & 6.1 & 9.2 & 9.7 & 2.2 \\
25 & 2.2 & 5.8 & 9.7 & 14.5 & 1.7 \\
30 & 2.3 & 5.0 & 9.6 & 8.5 & 3.1 \\
35 & 1.9 & 4.0 & 6.3 & 6.8 & 2.6 \\
40 & 1.8 & 3.3 & 4.8 & 7.4 & 2.0 \\
45 & 1.5 & 2.6 & 4.0 & 4.8 & 1.9 \\
\hline
\end{tabular}

Table S14. Average $C_{10} E_{6}$ aggregation numbers using radii generated by ratio of $\mathrm{D}_{\mathrm{C} 6 \mathrm{H} 12} / \mathrm{D}_{\mathrm{C} 10 \mathrm{E} 6}$ for solutions of $15 \%$ by mass polydisperse $\mathrm{C}_{10} \mathrm{E}_{6}$ in cyclohexane with indicated water content

\begin{tabular}{crrrrr}
\hline $\mathrm{T} /{ }^{\circ} \mathrm{Cl} \% \mathrm{H}_{2} \mathrm{O}$ & 0 & 1 & 2 & 3 & 4 \\
$W$ & 0.08 & 1.73 & 3.32 & 5.00 & 6.37 \\
\hline 10 & 17.0 & 82.7 & 240 & 696 & 2290 \\
15 & 18.9 & 82.7 & 168 & 859 & 983 \\
20 & 15.4 & 60.0 & 122 & 348 & 1741 \\
25 & 10.8 & 48.4 & 110 & 419 & 597 \\
30 & 10.8 & 39.1 & 112 & 185 & 653 \\
35 & 8.5 & 29.0 & 64.1 & 125 & 305 \\
40 & 7.9 & 21.2 & 40.6 & 105 & 149 \\
45 & 5.9 & 16.8 & 28.7 & 62.5 & 83 \\
\hline
\end{tabular}

